\title{
Origin and Specialization of Splenic Macrophages
}

A-Gonzalez, Noelia ${ }^{1 *}$ and Castrillo, Antonio ${ }^{2,3}$

${ }^{1}$ Institute of Immunology. University of Münster, 48149 Münster, Germany

${ }^{2}$ Instituto Investigaciones Biomédicas “Alberto Sols”, IIBM Madrid, Spain

Centro Mixto Consejo Superior de Investigaciones Cientificas y Universidad Autonoma de Madrid (IIBM CSIC-UAM).

${ }^{3}$ Unidad De Biomedicina (Unidad Asociada al CSIC), IIBM- Universidad Las Palmas de Gran

Canaria, ULPGC. Grupo de Investigación en medio ambiente y Salud (GIMAS), Instituto

Universitario de Investigaciones Biomedicas y Sanitarias (IUIBS, ULPGC).

* Corresponding Author: alonsogo@uni-muenster.de 


\begin{abstract}
Macrophage heterogeneity in the spleen has been long documented, with four subsets populating the different splenic compartments. The diverse environments on the splenic compartments determine their varied phenotype and functions. In the white pulp, highly phagocytic macrophages contribute to the generation of the immune response. The marginal zone contains two populations of macrophages, which also contribute to the immune response. Their strategic position in the bloodstream and their unique phenotype confer them a crucial role in the defense against blood borne pathogens, placing them at the crossroad between innate and adaptive immune responses. Macrophages in the red pulp are classically linked to homeostatic and metabolic functions in erythrocyte phagocytosis and iron recycling. We review here recent advances demonstrating the importance of macrophage ontogeny and organ development in determining the phenotype, transcriptional profile and, ultimately, the functions of the populations of splenic macrophages.
\end{abstract}




\section{Introduction}

Composed by both connective and lymphoid tissue, the spleen is a complex organ with a highly organized compartmentalization and an intricate microcirculatory system [1]. The particular microanatomy of the murine spleen makes it a unique organ which harbors heterogeneous populations of stromal, endothelial and immune cells. In the red pulp, venous sinusoids are mixed with leukocytes, stromal cells and splenic cords creating a network that allows the selection of effete red blood cells and unwanted material by professional phagocytes [2]. The white pulp is a dense lymphoid tissue compartment in which B cells are organized in follicles, surrounded by $\mathrm{T}$ cell areas, where the immune response and the generation of antibodies take place within the germinal centers [1]. The marginal zone of the murine spleen is formed by a sinusoid system of sinus lining cells that surround the white pulp, where the blood freely percolates on its way to the red pulp. These areas were believed to be transitional zones, with migrating populations, however, in the mouse spleen, several unique populations of macrophages, B cells and endothelial cells are permanent residents of the marginal zone [3]. Hence, to understand the variety of immune cell types in the different compartments of the spleen, the coexisting microenvironments must be considered. Those specific environmental cues ensure the multifaceted mechanisms that allow the spleen to perform different immune and homeostatic functions. Similarly, the concomitant microenvironmental signals potentially influence the different immune cell populations found in the various compartments of the murine spleen. In this review we will focus on the variety of splenic resident macrophages, with a particular emphasis on the murine system. A simple definition of the term "macrophage" is currently a challenge due to the continuous advances in the field, with the definition of their multiple origins $[4,5,6,7]$, the study of their plasticity [8] and reprogramming capacities during disease [9], and with the characterization of novel subsets [10,11]. Macrophages are yet considered a myeloid cell type, together with granulocytes, monocytes and dendritic cells. All myeloid linages were classically defined as hematopoietic cells that derive from a single clonogenic common myeloid progenitor [12]. This seminal report described a common myeloid progenitors that gave rise to exclusive megakaryocyte/erythrocyte or granulocyte/macrophage progenitors and subsequently differentiated into erythrocytes, granulocytes, monocytes, dendritic cells (DCs) and macrophages. While for some time these progenitors were believed to give rise to all tissue myeloid cells subsets, it is now accepted that the generation of some tissue resident macrophages occurs before the establishment of definitive hematopoiesis and develop independently of the common myeloid progenitors of the bone marrow [4, 5, 6, 7]. As 
mentioned above, in the last decade, a prolific literature has been released about the origin and establishment of tissue resident macrophages, implicating multiple factors, such as transcriptional regulators, microenvironment and plasticity (recently reviewed in $[13,14,15]$ ). Many efforts have been done to characterize the myeloid cell subsets in the spleen, both in the steady state and during disease. Due to the intrinsic antigen presentation capabilities and migratory behavior of many myeloid cells, a plethora of these subsets can be identified in the spleen under different circumstances, substantially complicating the scenario [16]. In addition, the aforementioned coexisting microenvironments in the spleen create the perfect niches for the homing of heterogeneous myeloid populations into the different compartments. In fact, four different populations of resident macrophages are found in the three compartments of the murine spleen [17]. Hence, it perfectly resembles the influence of the microenvironment on tissue-resident macrophage heterogeneity in a single organ, through diverse macrophage adaptations to each compartment. We will review here the ontogeny, phenotypic profile and functional specialization of the different macrophage subsets of the murine spleen with a special focus on the influence of the microenvironment in their transcriptional activity.

\section{Cellular and molecular mechanisms that regulate splenic development}

As a secondary lymphoid organ, certain aspects during the splenic embryonic and early postnatal development are common to lymph node organogenesis. The initiation of lymphoid organogenesis requires the spatiotemporal coordinated action of cytokines, chemokines, and transcription and angiogenic factors [18]. The interplay of cytokines (importantly lymphotoxin- $\alpha(\mathrm{LT} \alpha)$ ), chemokines (e.g. CXCL13), transcription factors (e.g. ROR $\gamma$ ) occurs mainly within two cell types: a subtype of innate lymphoid cell, known as lymphoid tissue initiating cell (LTi), and the stromal lymphoid tissue organizer cells (LTo) [19, 20]. On the first stages of lymphoid organogenesis, retinoic acid (RA) is produced by nerve fibers and induces the expression of CXCL13 in mesenchymal cells that, in turn, attract LTi cells to form the organ anlage [21]. These cells produce high amounts of LT $\alpha$ and $\beta$, which activate LT $\beta$ R signaling pathways in LTo cells, promoting the expression of cytokines, chemokines and adhesion molecules crucial for the fully organization and maturation of the lymph nodes [22]. These events, together with the exposure to the external environment after birth, complete the maturation of the lymphoid organs. Although the previously mentioned sequence of events applies to the majority of secondary lymphoid organs, some differences on the molecules involved, specific signaling pathways and temporal events have been described for Peyer's Patches (PPs) and spleen development. For example, a recent study shows that 
lymph node, but not PPs or spleen, organogenesis is dependent on LTi-cell-mediated activation of lymphatic endothelial cells, instead of mesenchymal LTo, which is a subsequent event [23]. Nonetheless, as mentioned above, the spleen is a particular case among secondary lymphoid organs, due to their multiple functions, including their ability to act as an hematopoietic organ during development, and even in special occasions in the adult life. These factors influence its organogenetic process and certainly impact the establishment of the myeloid and lymphoid populations. Particularly, the definitive compartmentalization and establishment of the splenic resident macrophages are complete between the $3^{\text {rd }}$ to $4^{\text {th }}$ week of the postnatal development [24].

The spleen develops from the mesoderm, starting at embryonic day E12 in mice, with the appearance of the splanchnic mesodermal plate, considered as the splenic anlage[1]. Several transcription factors have been shown to be crucial for these first steps on splenic development, such as Pbx1, Hox11 (Tlx1), Nkx3.2 (Bapx1) and Pod1 (capsulin, Tcf21) [25]. Particularly, Tlx 1 is crucial to regulate RA signaling in the developing spleen [26]. Around day E13.5-E14 LTi cells can be found in the spleen, suggesting similarities with the initial developmental steps of the lymph nodes. However, these cells do not express LT $\alpha$ in the neonatal spleen and only B cells at 4 days after birth do, underlying different pre- and postnatal developmental pathways in the spleen [27]. Although in an LT $\alpha \beta$ independent manner, splenic neonatal LTi are believed to contribute to the white and red pulp segregation through their interaction with MAdCAM-1+CD31+CD201+ spleen stromal organizer cells [28]. In contrast, in the adult spleen, LT $\alpha \beta$ producing LTi cells were reported to be crucial for the maintenance or B and T cell compartmentalization through their interaction with LT $\beta R$ expressing endothelial cells [29]. Nevertheless, during cytomegalovirus (CMV) and lymphocytic choriomeningitis virus infections, both of which cause disruption of the $\mathrm{B}$ and $\mathrm{T}$ cell compartmentalization, the maintained expression of lymphoid chemokines needed for $\mathrm{B}$ and T cell areas recovery occurred through LT-independent pathways, suggesting a reactivation of the embryonic program under stress conditions [30, 31, 32]. Hence, the different developmental and organizational pathways during embryonic and postnatal phases must be considered so as to understand the establishment of the different cell populations in the spleen. Remarkably, different macrophage subsets populate the murine spleen at different stages during embryonic and postnatal development, following the compartmentalization and structural events of the different areas. . Indeed, macrophages that resemble the red pulp macrophage population by their expression of F4/80 are present since embryonic stages but only locate in the red pulp between postnatal day 14-21, when the 
definitive red and white pulp segregation is patent; marginal zone macrophages (MZMs) and marginal metallophilic macrophages (MMMs) appear only after birth, around postnatal day 714, and are located at their areas between days 21-28 after birth [24]; and tingible body macrophages (TBMs) are only visible in the white pulp once the germinal centers are active through their contact with the external environment after weaning (Fig. 1). Therefore, the ontogeny and development of the different resident macrophage populations of the spleen is also influenced by the various mechanisms of splenic development, ultimately influenced by the microenvironment. The few animal models available with myeloid-specific deficiencies in molecules implicated in spleen development reveal mild alterations on spleen development. However, the expression of these ontogenic factors in macrophages seems to be important for the maintenance of the splenic adult structure. Remarkably, a work from Mountz and collaborators in 2015 addressed the contribution of myeloid cell derived LT $\beta$ R to macrophage establishment in the marginal zone. The study shows that macrophages in the marginal zone of the spleen were fully developed one month after birth but suffer slight disruption as they age. Yet, a correct compartmentalization of the spleen was observed in these mice. The authors also described an increased germinal center reaction in aged $L t b r^{f l f l} L y s-C r e$, probably due to the accumulation of apoptotic cell-derived antigens in the marginal zone as a consequence of MZM disruption over time [33]. Altogether, these data suggest that, although the correct establishment of macrophages in the spleen occurs in parallel with organ development, macrophage-derived factors seem to be dispensable for the correct splenic organogenesis. In addition, analysis of the recombination efficiency of several Cre- myeloid drivers, using several mouse strains, showed a low recombination efficiency of the Lys-Cre promoter, in spleen macrophages, indicating that the Loxp/Lys-Cre system might not be ideal, at least under steady-state conditions, to study the functions of splenic macrophages [34].

\section{Functional specialization of macrophages in the spleen}

As previously exposed, immense advancements in the molecular definition of tissue-resident macrophages, based on their ontogeny and microenvironment, have been done. Yet, the homeostatic and immunological significance of all these concepts in selected macrophage populations in specific niches need further investigation. One of the current challenges is to define whether specific macrophage functions, are dictated by their ontogeny, at specific locations and whether tissue-resident macrophages can be reprogrammed by novel environments in the adult. The current scenario requires further investigations; yet, some evidences already underlie a crosstalk between macrophages and tissue microenvironment in 
the adult that shapes macrophage functions and contribute to organ integrity [35]. Particularly intriguing is the case of the spleen, with the multiple scenarios and macrophage subsets that it harbors. Here we give an overview of the trophic and immunological functions of the splenic macrophage populations connected to their ontogeny and tissue microenvironmental cues.

\section{1. "Metabolic": Red Pulp Macrophages}

Red pulp macrophages most known function is the clearance of effete red blood cells that are trapped within the red pulp sinusoids and stromal network. These cells reside exclusively in the red pulp and are identified as F4/80+VCAM1+CD11b ${ }^{\text {lo }}$ by flow cytometry. The molecular mechanisms by which senescent erythrocytes are recognized by RPMs are not completely understood. They are, nonetheless, equipped with the adequate machinery to process and metabolize heme and Iron after senescent erythrocyte clearance. High levels of expression of the scavenger receptor CD163, Spi-C, VCAM1, Heme Oxigenase (HO-1) and ferroportin, by RPMs ensure their regulation of iron metabolism [36].

The metabolic function of RPMs is clearly influenced by the environment and linked to the transcriptional regulation of their developmental pathways. RPMs develop in mice during embryonic and fetal stages, with very few, if any contribution of monocytes. However, after macrophage depletion, bone marrow monocytes are able to repopulate the red pulp with F4/80+VCAM1+ macrophages [37]. RPMs were shown to derive partially from yolk sac macrophages [38], with the contribution of fetal definitive hematopoiesis [39]. However, the precise fetal progenitor from which RPM derive is not completely determined.

Heme regulates the expression of Spi-C, a transcription factor responsible for the development of RPMs. Spi-C, subsequently regulates the expression of VCAM1, influencing as well in iron metabolism. However, the expression of HO-1 and ferroportin seem to be directly influenced by Heme and independent of Spi-C [40]. Ultimately, the crosstalk between erythrophagocytosis, iron recycling and RPM transcriptional activation is needed to the maintenance of these populations in the adult spleen. Numerous evidences point to the existence of a different mechanism of damaged erythrocyte elimination, through a process called eryptosis that involves exposure of phosphatidylserine in the outer membrane and shrinking of the cell, in an analogous fashion that nucleated cells suffer apoptosis [41]. These eryptotic erythrocytes are, however, believed to be recognized and eliminated by different subsets of phagocytes in the spleen [42]. 
RPMs have also been shown to perform immunological functions, in response to inflammatory stimuli and parasite infections. Using Spic-/-, Kim and colleagues showed that RPMs produce high amounts of type I interferons, playing a key role in the defense against Plasmodium chabaudi [43]. In vitro studies using isolated RPM in co-cultures with CD4+ T cells have revealed an implication of RPMs in the process of differentiation or T cells towards regulatory T cells [44].

\section{2. "Phagocytic": White Pulp Macrophages}

The white pulp has for many years been considered as the immunological compartment of the spleen. Now we know that the red pulp also perform some immunological functions and the marginal zone is as well a dynamic area where specific immune responses take place. Within the germinal centers, $\mathrm{F} 480{ }^{\text {neg }} \mathrm{CD} 68+$ macrophages containing phagocytosed apoptotic cells are frequently found. Condensed chromatin fragments are visible inside these macrophages displaying a dark blue staining pattern when using hematoxylin and eosin. Consequently, these macrophages were originally called tingible body macrophages (tingible meaning stainable). TBMs are analogous to macrophages found in the germinal centers of lymph nodes and PPs, as well as in the thymus of adult mice, during its physiological involution. In the spleen, their main function is the phagocytosis of apoptotic B cells generated in the germinal centers during antigenic responses, where hyper proliferation and somatic hypermutation of these cells take place [45]. In addition, germinal centers are also the sites of B cell selfrenewal and clonal selection, crucial to maintain tissue homeostasis. During the high affinity maturation of the clonal selection process, autoreactive B cells are also eliminated by apoptosis and cleared by TBMs. Hence, the highly phagocytic macrophages in the white pulp play a dual role as homeostatic regulators and actively participating of the immune responses against antigens.

Certainly, the white pulp environment shapes the phagocytic aspect of these macrophages. Several soluble molecules required by the macrophage to engage the apoptotic cell during its recognition phase are highly expressed in the splenic white pulp, such as Mfge8, Gas6, or C1q. TBMs as well express high levels of phagocytic receptors as compared with the other macrophage populations in the spleen, for example, CD68, Mertk, Timd4 or CD36 [17]. All these molecules have been previously shown to play decisive roles in the phagocytosis of apoptotic cells, however, whether their elevated expression in this macrophage subset is influenced by specific white pulp derived signals is still unclear. Nonetheless, we have 
previously shown that the constant feeding of macrophages with apoptotic cells promote the expression of Mertk, Gas6 and CD36 [46]. Hence, one possible scenario is that the constant phagocytosis of B cells in the germinal centers provides the signals to shape the phenotype and function of TBMs, contributing to maintain peripheral B tolerance and regulating the germinal center reaction, ultimately, ensuring the correct defense against antigen-driven inflammatory processes.

\section{3. "Immune": Marginal Zone Macrophages}

The marginal zone of the murine spleen is a dynamic structure located between the red and white pulps where the blood is shed before percolating to the red pulp. Its specific location and cell composition create an adequate environment for the recognition and clearance of blood borne antigens. In addition, it is the starting point in the generation of the immune response against $\mathrm{T}$ cell independent antigens. The marginal zone contains a special stromal endothelial cell, called the marginal sinus lining cell that expresses MadCAM1. In contrast to MadCAM1+ endothelial cells in the lymph nodes, splenic marginal sinus lining cells do not form blood or lymphatic vessels, but they line up forming an outer ring around the white pulps. Closely associated to these endothelial cells, concentrate the CD169+ MMMs and outer, several layers of MZMs coexist with specialized B cells and dendritic cells, supported by a network of stromal conduits. Remarkably, the marginal zone is the only microanatomical environment in which two phenotypically and functionally different macrophage populations coexist. Both populations of macrophages are responsible for the clearance of blood-borne encapsulated bacteria, such as meningococci [47], Campylobacter jejuni [48] or Streptococus pneumoniae [49]. They have also been shown to phagocytose parasites, like Leishmania donovani [50]. Copious speculations about their separate functions were made in the past, still being a controversial topic. Conditional depletion of MMM or MZM via diphtheria toxin receptor, using $C d 169^{D T R}$ and $C d 11 c^{D T R}$ transgenic mice, showed no conclusive results on their selectivity of splenic macrophage depletion [51]. Another caveat in the study of these populations is the difficulty to isolate them and the lack of mouse models deficient specifically on one of them. The isolation of macrophages of the marginal zone still remains a challenge due to their small frequency in the spleen, their close contact with endothelial and stromal cells and the particular properties of their surface markers. MMMs and MZMs are defined by the high expression of the lectins CD169, Sialoadhesin, and SIGNR1 respectively. These lectins get rapidly internalized by the macrophage during sample preparation for flow 
cytometry or sorting techniques, which hinders the application of high throughput techniques to define their transcriptomic profile. Nevertheless, different functions have been proposed for these two macrophage populations:

\subsubsection{Marginal Metallophilic macrophages}

Their high expression of CD169, Sialoadhesin, similar to the subcapsular sinus macrophages in the lymph nodes suggest analogous functions, however the different disposition of both populations in the organs and the particular anatomy and environmental cues of the spleen confers them unique features.

MMMs have been selectively implicated in the degradation and clearance of viruses, such as porcine reproductive and respiratory syndrome virus [52]. An elegant study from den Haan and colleagues showed that CD169+ MMMs specifically induced T cell responses. Using conjugated OVA-antibodies to target different antigen presenting cells in the spleen, the authors demonstrated that MMM can specifically take up antigens, transfer them to CD8+ dendritic cells, ultimately activating T cells [53], showing their potential as antigen presenting cells and their direct implication in the generation of the adaptive immune response. Besides their immunological functions on the recognition of blood-borne pathogens and antigens, processes dictated by their surrounding microenvironment, MMMs also perform trophic functions by actively interacting with their neighboring cells. Follicular B cells are essential to the maintenance of MMMs through the release of LT $\alpha \beta$ [54]. In addition, follicular B cells have been shown to migrate towards the marginal zone, in response to oxysterols, suggesting the idea that MMMs are producing those oxysterols [55]. Together with the fact that $\operatorname{LXR} \alpha$, a transcription factor activated by oxysterols, drives the differentiation of MMMs, this would potentially contribute to understand how metabolic pathways actively influence the differentiation of macrophages in the marginal zone [24]. The close association of MMMs with the MadCAM1+ endothelial sinus lining cells suggests a cooperative role for these macrophages in the organizing functions of the sinus lining cells in the maintenance of the splenic structure. This was in principle supported by evidences of mouse models lacking MMM, which also showed disrupted splenic compartmentalization, with disorganized B cell follicles [56]. However, we showed in LXR $\alpha-/-$ mice, where there is total absence of cd169+ MMM, no apparent affection of sinus lining cells and a proper organization of the B and T cell areas [24], ruling out an organizational function for these macrophages. 


\subsubsection{Marginal zone macrophages}

The immunological role of MZMs is highly determined by the nature of the antigens that they phagocyte from the blood stream and their close interaction with MZ-B cells. These specialized B cells recognize blood-borne antigens and migrate to the follicles in the white pulps to present them to FDCs, which ultimately generate the germinal center reaction. SIGNR1 expressed by the MZMs has revealed as a key molecule in the interaction between MZ-B cells and MZMs. In the absence of MZ-B cells there is a downregulation of SIGN-R1 in MZMs [57] and, reciprocally, when SIGN-R1 is not present, the migration of MZ-B cells to the follicles is impaired, therefore, altering their interaction with FDCs and germinal center reaction [24, 58]. Moreover, the scavenger receptor MARCO, which is also highly expressed by MZMs, had been previously shown to interact with B cells promoting their maintenance and correct positioning in the marginal zone [59]. This crosstalk between the MZMs and MZ$\mathrm{B}$ cells is crucial to ensure the correct immune responses against $\mathrm{T}$ cell independent antigens, contributing to maintain central tolerance. Furthermore, it is crucial to understand MZM intrinsic plasticity in response to environmental cues. Using the LXR $\alpha-/-$ model, we observed that in the absence of MZMs clearance of blood borne pathogens was taken over by RPMs, underlying the plasticity of those macrophages. However, these mice exhibited a blunted immune response, with a decreased generation of specific antibodies IgM antibodies, against T cell independent antigens, suggesting that physical communication with MZ-B cells is still needed to generate the immune response. These results suggest that, although splenic macrophages possess the ability of reprogramming some of their functions, the interplay with the environment is crucial to determine the specific functions of the different macrophage subsets.

MZMs are also implicated in central tolerance through phagocytosis of blood-borne apoptotic cells. Several studies showed that intravenously injected apoptotic cells were rapidly phagocytosed by MZMs $[60,61]$. This prompted the suppression of an immune response against dying cell associated antigens, leading to the maintenance of immune tolerance. Interestingly, the interaction of LT $\alpha \beta$ expressed by MZ B cells and LT $\beta$ R expressed by MZMs, was shown to be important for efficient engulfing of circulating apoptotic cells by MZMS in lupus disease [33]. Remarkably, these studies underlie the importance of MZMs in the control against developing autoimmune diseases, in a similar fashion as of TBMs. In both cases, the molecules implicated in this process are not fully identified; however the different 
origin of the cells that they phagocytose may lead to different transcriptional program on each macrophage population, raising the heterogeneous properties of splenic macrophages.

Supporting this theory, in a recent report, we showed that different environments contribute to increase tissue-resident macrophage heterogeneity through the phagocytosis of unwanted circulating cells [11].

\section{Transcriptional landscape of splenic macrophages origin}

Tissue resident macrophages show diverse origins and differentiation pathways influenced by the environment, which impacts their transcriptional profile and activation landscapes [13, 39]. The integration of single-cell RNA and ATAC (assay for transposase-accessible chromatin) sequencing (RNA-seq and ATAC-seq), cytometry by time-of-flight mass spectrometry (CyTOF), genetic fate mapping, and functional analyses using cutting edge techniques, such as CRISPR-Cas9, enormously contributes to a deep phenotypic characterization of the diverse tissue resident macrophage populations. This is now basic to understand their differentiation and activation, also during disease [15]. Over the last few years, huge advances have been made to define the transcriptional programs of macrophages during development, which has contributed as well to understand the influence of the microenvironment in their reprogramming capacities [8]. Several transcription factors have been described as part of a common macrophage core signature during developmental phases. Multipotent erythro-myeloid progenitors (EMPs) expressing the transcription factor $c-M y b$ are found in the embryo at day E8.5 and were shown to give rise adult tissue resident macrophages through primitive and definitive hematopoiesis [62]. To define the complete cascade of transcriptional events in macrophages, several studies explored the genome-wide locations of enhancers and master regulators of myeloid precursors and macrophages. PU.1 is considered as a master regulator of macrophages occupying most enhancers [63]. Maf and $M a f b$ transcription factors are also implicated in the regulation of macrophage differentiation and proliferation [8]. In addition to these transcriptional master regulators, different macrophage populations are specifically dependent on transcription factors for their development and differentiation. For example, the transcription factor Gata6 is essential for the differentiation of peritoneal macrophages [64] and the nuclear receptor PPAR $\gamma$ is crucial for alveolar macrophage identity [65]. Particularly, in the spleen, Spic and IRF8 regulate the development of red pulp macrophages [66-68] and $L X R \alpha$ of marginal zone and metallophilic macrophages [24]. 
Whether organogenesis influences macrophage development or vice versa on each particular organ is still an open debate in the field. A report in Science in 2016 suggests that macrophage differentiation is an integral part of organogenesis, based on the description of a novel macrophage precursor, pre-macrophages or pMacs, that colonizes the whole embryo at E9.5 and ultimately specify into tissue-resident macrophages in the different organs that they study [69]. Yet enlightening and intriguing, this study does not include adult splenic macrophages in the screening; hence still the question of whether the four splenic macrophage populations share a common precursor and primitive transcriptional profile remains unknown. As mentioned above, several transcription factors have been described to regulate the development of three splenic macrophage subsets:

\subsection{Spi-C and IRF8: red pulp macrophages}

Spi-C was the first transcription factor identified that controls the development of RPMs. Murphy and colleagues showed a dramatic reduction of RPMs in Spic-/- mice with almost no affection of other macrophage populations in the spleen [65]. The authors showed that Spi-C played a cell-autonomous effect in the development of RPMs, since it was corrected by retroviral Spi-C expression in reconstitution studies through bone marrow transplant. Spi-C expression is controlled by heme through the degradation of its transcriptional repressor BACH1 [70]. Spic-/- mice accumulate large amounts of heme and iron in their splenic red pulp, therefore showing splenomegaly and defects in iron metabolism. Interestingly, the same group later showed that Spic was also implicated in the development and maintenance of bone marrow F480+VCAM1+ macrophages, a subtype of bone marrow resident cells with ironrecycling capacities similar to RPMs, also influenced via heme and BACH1 regulation [70]. Remarkably, these studies demonstrate a direct link between Spi-C-dependent transcriptional activity and iron metabolism in the development of splenic and bone marrow macrophages. There are, nonetheless, several interesting unexplored paths regarding Spi-C activity. For example, the direct Spi-C downstream targets that drive the differentiation of monocytes into RPMs are still not well-defined. Studies that explore the genome-wide Spi-C binding locations, along with RNA-seq experiments would possibly shed light on these questions. The role of other transcription factors, such as IRF4 and IRF8, implicated in the development of multiple hematopoietic cells (for example CD11b+ dendritic cells [71] and B cells [68, 72] respectively) has been reported to be important for the development of RPMs. Indeed, the lack of both factors causes a dramatic decrease of the RPM population [68]. 


\subsection{LXR $\alpha$ : macrophages in the marginal zone}

Despite the technical hitches in the isolation of marginal zone macrophages explained in point 3.3., the combination of imaging techniques and in vivo functional analysis with the use of diverse mouse models, allow us and others to define crucial molecules for their development and establishment. In the late nineties, with the generation of different knock-out and transgenic mouse models of the lymphotoxin (LT)/tumor necrosis factor (TNF) family, the implication of these cytokines on the development of marginal zone macrophage populations was evidenced (reviewed in [56]). However, the mouse models lacking both populations of marginal zone macrophages, LT $\alpha-/-$, LT $\beta-/-$, and LT $\beta$ R-/-, showed also profound defects in secondary lymphoid organ development, with absent lymph nodes and PPs, and disorganized $\mathrm{B}$ and $\mathrm{T}$ cell zones in the spleen $[73,74]$. Hence, the specific role of these cytokines in macrophage development is not completely clear, and macrophage deficiencies are believed to be consequence of the organogenetic impairment observed in those animals.

To date, the only transcription factor described to be responsible for the development of macrophages in the marginal zone is the transcription factor LXR $\alpha$, a member of the nuclear receptor superfamily. We showed that $L x r \alpha-/-$ mice selectively lack MMMs and MZMs but showed RPMs and TBMs in their appropriate splenic locations. Remarkably, lymph node macrophages, defined by the expression of the same surface lectins, were not affected in these animals. In an effort to identify the specific progenitor of these macrophages, we performed different functional analysis using bone marrow chimeras, characterization of bone marrow progenitors and transferring isolated monocytes. We showed that adoptively transferred LXR $\alpha$-sufficient CX3CR $1{ }^{\text {int }}$ Ly6C ${ }^{\text {hi }}$ monocytes were able to populate the marginal zones of Lxr $\alpha$-/- mice and to differentiate into both macrophage subsets [24]. We concluded that, at least when the marginal zone space lacks macrophage populations in LXR $\alpha$-deficient mice, MMMs and MZMs can develop correctly from bone marrow monocyte precursors, pointing out to definitive hematopoiesis as responsible for the ontogeny of MZ- and MMmacrophages. Fully differentiated MMMs and MZMs start to appear in the postnatal murine spleen, and are only properly located in the marginal zone after weaning, likely ruling out primitive hematopoiesis as their primary source. However, to definitely exclude primitive macrophages as precursors of MZ macrophages in adult mice under homeostasis, additional studies using parabiosis experiments in WT and LXR-deficient mice will be required. Whether macrophages in the marginal zone might have a unique monocytic origin and whether the specific transcriptional pathway by which LXR $\alpha$ might be directly responsible for it still remains to be clarified. One possibility is that oxysterols, the endogenous ligands of 
LXR, in the spleen activate LXR $\alpha$ to drive the differentiation of monocytes to marginal zone macrophages. However, further studies should be done to clarify these hypotheses. Interestingly, several studies speculate that macrophages in the marginal zone might be producing oxysterols that also participate in the regulation of follicular B cell migration [55, 75].

\section{Conclusions and future perspectives}

The potential communication between the different populations of splenic macrophages has been proposed to be crucial for their development and functions. For example, the mouse models with marginal zone macrophage deficiencies normally lack both MMMs and MZMs, suggesting a strong interdependence between both populations in their developmental pathways. However, little is known about the potential communication between white and red pulp macrophages or whether there are common pathways during their development. The temporal differences in the establishment of the four populations in the spleen suggest different ontogeny and differentiation pathways. Whether there is a unique progenitor for all splenic macrophages remains to be clarified. Applying single-cell sequencing to myeloid progenitors at different stages together with the adult macrophage populations will be useful to stablish common pathways and transcription profiles, and it would contribute to build a "molecular map" of the development and differentiation of each population. However, the different splenic microenvironmental cues that influence the phenotypic landscape of the macrophage populations in the spleen, have only been partially described. Namely, the specific environmental cues crucial for marginal zone macrophage development still remain unknown. During spleen development, stromal cells act as central regulators, although it is still unknown which specific cues are involved in this process. Interestingly, a recent study shows that oxysterols produced by stromal fibroblastic cells promoted lymphoid tissue formation, by signaling through their receptor GPR183 expressed by LTi cells [76]. This finding underlies the importance of the communication of the microenvironment with precursors, to establish definitive organogenesis. Similarly, these molecules produced by stromal cells could directly influence macrophage precursors during their development and establishment. Whether oxysterols in the marginal zone specifically activates LXR $\alpha$ in monocytes conditioning the establishment of macrophage populations in that area is a possible mechanism that remains to be explored. 


\section{Figure Legends}

Figure 1. Ontogeny and Establishment of Splenic-Resident Macrophages. Macrophages in the red pulp originate from primitive yolk sac and fetal definitive hematopoiesis, the latest through fetal monocytes. In the postnatal spleen, red pulp macrophages are found since early stages dispersed throughout the yet non-compartmentalized neonatal spleen. Between postnatal day 7 and 14, primitive lymphoid follicles form and first marginal metallophilic macrophages appear in a disorganized manner. During the third week of the postnatal spleen development, white and red pulp compartments start to define, with the concomitant onset of the marginal zone macrophage populations. At the same time, red pulp macrophages are excluded from the white pulp areas and the first tangible body macrophages appear coinciding with the generation of the germinal center reaction in some follicles. At day 28 of the postnatal development, the spleen shows its definitive microanatomic structure with define white and red pulps and marginal zone. At this time, the four populations of macrophages inhabit their compartments. The scheme also shows the transcription factors implicated in the proper differentiation and establishment of red pulp macrophages (Spi-C and IRF8), their precursors (PU.1 and Myb) and marginal metallophilic and marginal zone macrophages (LXR $\alpha$ ). E: embryonic day; P: postnatal day; YS: yolk sac; FL: fetal liver; TB: tingible body; RP: red pulp; MZ: marginal zone; MM: marginal metallophilic; Mo: monocyte; MФ: macrophage. 


\section{Acknowledgments}

Research in Antonio Castrillo's lab is supported by grants from Spanish Ministerio de Ciencia y Tecnología [SAF2008-00057], Ministerio de Economia y Cometitividad [SAF2011-29244, SAF2014-56819-R, SAF2015-71878-REDT] and Comunidad de Madrid I+D [Grant S2010/BMD-2350 Raphyme-CM and B2017/BMD-3680 Chronobite-CM]. 


\section{References}

[1] R.E. Mebius, G. Kraal, Structure and function of the spleen, Nat. Rev. Immunol. 8 (2005) 606-616.

[2] B.S. Steiniger, Human spleen microanatomy: why mice do not suffice, Immunology. 3 (2015) 334-346.

[3] G. Kraal, R. Mebius, New insights into the cell biology of the marginal zone of the spleen, Int. Rev. Cytol. (2006) 175-215.

[4] C.C. Bain, A. Bravo-Blas, C.L. Scott, E.G. Perdiguero, F. Geissmann, S. Henri, B. Malissen, L.C. Osborne, D. Artis, A.M. Mowat, Constant replenishment from circulating monocytes maintains the macrophage pool in the intestine of adult mice, Nat. Immunol. 10 (2014) 929-937.

[5] F. Ginhoux, M. Greter, M. Leboeuf, S. Nandi, P. See, S. Gokhan, M.F. Mehler, S.J. Conway, L.G. Ng, E.R. Stanley, I.M. Samokhvalov, M. Merad, Fate mapping analysis reveals that adult microglia derive from primitive macrophages, Science. 6005 (2010) 841-845.

[6] G. Hoeffel, Y. Wang, M. Greter, P. See, P. Teo, B. Malleret, M. Leboeuf, D. Low, G. Oller, F. Almeida, S.H. Choy, M. Grisotto, L. Renia, S.J. Conway, E.R. Stanley, J.K. Chan, L.G. Ng, I.M. Samokhvalov, M. Merad, F. Ginhoux, Adult Langerhans cells derive predominantly from embryonic fetal liver monocytes with a minor contribution of yolk sacderived macrophages, J. Exp. Med. 6(2012) 1167-1181.

[7] J. Sheng, C. Ruedl, K. Karjalainen, Most Tissue-Resident Macrophages Except Microglia Are Derived from Fetal Hematopoietic Stem Cells, Immunity. 2 (2015) 382-393.

[8] Y. Lavin, D. Winter, R. Blecher-Gonen, E. David, H. Keren-Shaul, M. Merad, S. Jung, I. Amit, Tissue-resident macrophage enhancer landscapes are shaped by the local microenvironment, Cell. 6 (2014) 1312-1326.

[9] M. Schiwon, C. Weisheit, L. Franken, S. Gutweiler, A. Dixit, C. Meyer-Schwesinger, J.M. Pohl, N.J. Maurice, S. Thiebes, K. Lorenz, T. Quast, M. Fuhrmann, G. Baumgarten, M.J. Lohse, G. Opdenakker, J. Bernhagen, R. Bucala, U. Panzer, W. Kolanus, H.J. Grone, N. Garbi, W. Kastenmuller, P.A. Knolle, C. Kurts, D.R. Engel, Crosstalk between sentinel and helper macrophages permits neutrophil migration into infected uroepithelium, Cell. 3 (2014) 456-468.

[10] R. Tacke, I. Hilgendorf, H. Garner, C. Waterborg, K. Park, H. Nowyhed, R.N. Hanna, R. Wu, F.K. Swirski, F. Geissmann, C.C. Hedrick, The transcription factor NR4A1 is essential for the development of a novel macrophage subset in the thymus, Sci. Rep. (2015) 10055.

[11] N. A-Gonzalez, J.A. Quintana, S. Garcia-Silva, M. Mazariegos, A. Gonzalez de la Aleja, J.A. Nicolas-Avila, W. Walter, J.M. Adrover, G. Crainiciuc, V.K. Kuchroo, C.V. Rothlin, H. Peinado, A. Castrillo, M. Ricote, A. Hidalgo, Phagocytosis imprints heterogeneity in tissueresident macrophages, J. Exp. Med. 5 (2017) 1281-1296.

[12] K. Akashi, D. Traver, T. Miyamoto, I.L. Weissman, A clonogenic common myeloid progenitor that gives rise to all myeloid lineages, Nature. 6774 (2000) 193-197. 
[13] S. Gordon, A. Pluddemann, Tissue macrophages: heterogeneity and functions, BMC Biol. 1 (2017) 53-017-0392-4.

[14] F. Ginhoux, M. Guilliams, Tissue-Resident Macrophage Ontogeny and Homeostasis, Immunity. 3 (2016) 439-449.

[15] F. Ginhoux, J.L. Schultze, P.J. Murray, J. Ochando, S.K. Biswas, New insights into the multidimensional concept of macrophage ontogeny, activation and function, Nat. Immunol. 1 (2016) 34-40.

[16] Y.Y. Hey, J.K. Tan, H.C. O'Neill, Redefining Myeloid Cell Subsets in Murine Spleen, Front. Immunol. (2016) 652.

[17] J.M. den Haan, G. Kraal, Innate immune functions of macrophage subpopulations in the spleen, J. Innate Immun. 5-6 (2012) 437-445.

[18] S.A. van de Pavert, R.E. Mebius, New insights into the development of lymphoid tissues, Nat. Rev. Immunol. 9 (2010) 664-674.

[19] G. Eberl, S. Marmon, M.J. Sunshine, P.D. Rennert, Y. Choi, D.R. Littman, An essential function for the nuclear receptor RORgamma(t) in the generation of fetal lymphoid tissue inducer cells, Nat. Immunol. 1 (2004) 64-73.

[20] T.D. Randall, D.M. Carragher, J. Rangel-Moreno, Development of secondary lymphoid organs, Annu. Rev. Immunol. (2008) 627-650.

[21] van de Pavert S.A., Olivier B.J., Goverse G., Vondenhoff M.F., Greuter M., Beke P., Kusser K., Höpken U.E., Lipp M., Niederreither K., Blomhoff R., Sitnik K., Agace W.W., Randall T.D., de Jonge W.J., Mebius R.E., Chemokine CXCL13 is essential for lymph node initiation and is induced by retinoic acid and neuronal stimulation, Nat. Immunol. (2009) 1193-1199.

[22] A. Futterer, K. Mink, A. Luz, M.H. Kosco-Vilbois, K. Pfeffer, The lymphotoxin beta receptor controls organogenesis and affinity maturation in peripheral lymphoid tissues, Immunity. 1 (1998) 59-70.

[23] L. Onder, U. Morbe, N. Pikor, M. Novkovic, H.W. Cheng, T. Hehlgans, K. Pfeffer, B. Becher, A. Waisman, T. Rulicke, J. Gommerman, C.G. Mueller, S. Sawa, E. Scandella, B. Ludewig, Lymphatic Endothelial Cells Control Initiation of Lymph Node Organogenesis, Immunity. 1 (2017) 80-92.e4.

[24] N. A-Gonzalez, J.A. Guillen, G. Gallardo, M. Diaz, J.V. de la Rosa, I.H. Hernandez, M. Casanova-Acebes, F. Lopez, C. Tabraue, S. Beceiro, C. Hong, P.C. Lara, M. Andujar, S. Arai, T. Miyazaki, S. Li, A.L. Corbi, P. Tontonoz, A. Hidalgo, A. Castrillo, The nuclear receptor LXRalpha controls the functional specialization of splenic macrophages, Nat. Immunol. 8 (2013) 831-839.

[25] A. Brendolan, E. Ferretti, V. Salsi, K. Moses, S. Quaggin, F. Blasi, M.L. Cleary, L. Selleri, A Pbx1-dependent genetic and transcriptional network regulates spleen ontogeny, Development. 13 (2005) 3113-3126. 
[26] E. Lenti, D. Farinello, K.K. Yokoyama, D. Penkov, L. Castagnaro, G. Lavorgna, K. Wuputra, L.L. Sandell, N.E. Tjaden, F. Bernassola, N. Caridi, A. De Antoni, M. Wagner, K. Kozinc, K. Niederreither, F. Blasi, D. Pasini, G. Majdic, G. Tonon, P.A. Trainor, A. Brendolan, Transcription factor TLX1 controls retinoic acid signaling to ensure spleen development, J. Clin. Invest. 7 (2016) 2452-2464.

[27] M.F. Vondenhoff, G.E. Desanti, T. Cupedo, J.Y. Bertrand, A. Cumano, G. Kraal, R.E. Mebius, R. Golub, Separation of splenic red and white pulp occurs before birth in a LTalphabeta-independent manner, J. Leukoc. Biol. 1 (2008) 152-161.

[28] J.K. Tan, T. Watanabe, Stromal Cell Subsets Directing Neonatal Spleen Regeneration, Sci. Rep. (2017) 40401.

[29] M.Y. Kim, F.M. McConnell, F.M. Gaspal, A. White, S.H. Glanville, V. Bekiaris, L.S. Walker, J. Caamano, E. Jenkinson, G. Anderson, P.J. Lane, Function of CD4+CD3- cells in relation to B- and T-zone stroma in spleen, Blood. 4 (2007) 1602-1610.

[30] E. Scandella, B. Bolinger, E. Lattmann, S. Miller, S. Favre, D.R. Littman, D. Finke, S.A. Luther, T. Junt, B. Ludewig, Restoration of lymphoid organ integrity through the interaction of lymphoid tissue-inducer cells with stroma of the T cell zone, Nat Immunology. (2008) 667-675

[31] C.A. Benedict, C. Trez, K. Schneider, S.Ha, G. Patterson, C.F. Ware, Specific Remodeling of Splenic Architecture by Cytomegalovirus PLoS Pathog. (2006) 2(3): e16

[32] N.H. Ruddle, E.M. Akirav, Secondary lymphoid organs: responding to genetic and environmental cues in ontogeny and the immune response, J. Immunol. 4 (2009) 2205-2212.

[33] H. Li, Y.X. Fu, Q. Wu, Y. Zhou, D.K. Crossman, P. Yang, J. Li, B. Luo, L.M. Morel, J.H. Kabarowski, H. Yagita, C.F. Ware, H.C. Hsu, J.D. Mountz, Interferon-induced mechanosensing defects impede apoptotic cell clearance in lupus, J. Clin. Invest. 7 (2015) 2877-2890.

[34] C.L. Abram, G.L. Roberge, Y. Hu, C.A. Lowell, Comparative analysis of the efficiency and specificity of myeloid-Cre deleting strains using ROSA-EYFP reporter mice, J. Immunol. Methods. (2014) 89-100.

[35] D. Hashimoto, A. Chow, C. Noizat, P. Teo, M.B. Beasley, M. Leboeuf, C.D. Becker, P. See, J. Price, D. Lucas, M. Greter, A. Mortha, S.W. Boyer, E.C. Forsberg, M. Tanaka, N. van Rooijen, A. Garcia-Sastre, E.R. Stanley, F. Ginhoux, P.S. Frenette, M. Merad, Tissue-resident macrophages self-maintain locally throughout adult life with minimal contribution from circulating monocytes, Immunity. 4 (2013) 792-804.

[36] T. Ganz, Macrophages and Iron Metabolism, Microbiol. Spectr. 5 (2016)

10.1128/microbiolspec.MCHD-0037-2016.

[37] C. Schulz, E. Gomez Perdiguero, L. Chorro, H. Szabo-Rogers, N. Cagnard, K. Kierdorf, M. Prinz, B. Wu, S.E. Jacobsen, J.W. Pollard, J. Frampton, K.J. Liu, F. Geissmann, A lineage of myeloid cells independent of Myb and hematopoietic stem cells, Science. 6077 (2012) 8690 . 
[38] S. Epelman, K.J. Lavine, A.E. Beaudin, D.K. Sojka, J.A. Carrero, B. Calderon, T. Brija, E.L. Gautier, S. Ivanov, A.T. Satpathy, J.D. Schilling, R. Schwendener, I. Sergin, B. Razani, E.C. Forsberg, W.M. Yokoyama, E.R. Unanue, M. Colonna, G.J. Randolph, D.L. Mann, Embryonic and adult-derived resident cardiac macrophages are maintained through distinct mechanisms at steady state and during inflammation, Immunity. 1 (2014) 91-104.

[39] Y. Lavin, A. Mortha, A. Rahman, M. Merad, Regulation of macrophage development and function in peripheral tissues, Nat. Rev. Immunol. 12 (2015) 731-744.

[40] C. Delaby, N. Pilard, H. Puy, F. Canonne-Hergaux, Sequential regulation of ferroportin expression after erythrophagocytosis in murine macrophages: early mRNA induction by haem, followed by iron-dependent protein expression, Biochem. J. 1 (2008) 123-131.

[41] F. Lang, E. Lang, M. Foller, Physiology and pathophysiology of eryptosis, Transfus. Med. Hemother. 5 (2012) 308-314.

[42] A. Larsson, A. Hult, A. Nilsson, M. Olsson, P.A. Oldenborg, Red blood cells with elevated cytoplasmic $\mathrm{Ca}(2+)$ are primarily taken up by splenic marginal zone macrophages and CD207+ dendritic cells, Transfusion. 7 (2016) 1834-1844.

[43] C.C. Kim, C.S. Nelson, E.B. Wilson, B. Hou, A.L. DeFranco, J.L. DeRisi, Splenic red pulp macrophages produce type I interferons as early sentinels of malaria infection but are dispensable for control, PLoS One. 10 (2012) e48126.

[44] D. Kurotaki, S. Kon, K. Bae, K. Ito, Y. Matsui, Y. Nakayama, M. Kanayama, C. Kimura, Y. Narita, T. Nishimura, K. Iwabuchi, M. Mack, N. van Rooijen, S. Sakaguchi, T. Uede, J. Morimoto, CSF-1-dependent red pulp macrophages regulate CD4 T cell responses, J. Immunol. 4 (2011) 2229-2237.

[45] N.S. De Silva, U. Klein, Dynamics of B cells in germinal centres, Nat. Rev. Immunol. 3 (2015) 137-148.

[46] N. A-Gonzalez, S.J. Bensinger, C. Hong, S. Beceiro, M.N. Bradley, N. Zelcer, J. Deniz, C. Ramirez, M. Diaz, G. Gallardo, C.R. de Galarreta, J. Salazar, F. Lopez, P. Edwards, J. Parks, M. Andujar, P. Tontonoz, A. Castrillo, Apoptotic cells promote their own clearance and immune tolerance through activation of the nuclear receptor LXR, Immunity. 2 (2009) $245-258$.

[47] C. Jones, M. Virji, P.R. Crocker, Recognition of sialylated meningococcal lipopolysaccharide by siglecs expressed on myeloid cells leads to enhanced bacterial uptake, Mol. Microbiol. 5 (2003) 1213-1225.

[48] A.P. Heikema, M.P. Bergman, H. Richards, P.R. Crocker, M. Gilbert, J.N. Samsom, W.J. van Wamel, H.P. Endtz, A. van Belkum, Characterization of the specific interaction between sialoadhesin and sialylated Campylobacter jejuni lipooligosaccharides, Infect. Immun. 7 (2010) 3237-3246.

[49] Y.S. Kang, J.Y. Kim, S.A. Bruening, M. Pack, A. Charalambous, A. Pritsker, T.M. Moran, J.M. Loeffler, R.M. Steinman, C.G. Park, The C-type lectin SIGN-R1 mediates uptake of the capsular polysaccharide of Streptococcus pneumoniae in the marginal zone of mouse spleen, Proc. Natl. Acad. Sci. U. S. A. 1 (2004) 215-220. 
[50] P.M. Gorak, C.R. Engwerda, P.M. Kaye, Dendritic cells, but not macrophages, produce IL-12 immediately following Leishmania donovani infection, Eur. J. Immunol. 2 (1998) 687695.

[51] S. Gordon, A. Pluddemann, S. Mukhopadhyay, Sinusoidal immunity: macrophages at the lymphohematopoietic interface, Cold Spring Harb Perspect. Biol. 4 (2014) a016378.

[52] N. Vanderheijden, P.L. Delputte, H.W. Favoreel, J. Vandekerckhove, J. Van Damme, P.A. van Woensel, H.J. Nauwynck, Involvement of sialoadhesin in entry of porcine reproductive and respiratory syndrome virus into porcine alveolar macrophages, J. Virol. 15 (2003) 8207-8215.

[53] R. Backer, T. Schwandt, M. Greuter, M. Oosting, F. Jungerkes, T. Tuting, L. Boon, T. O'Toole, G. Kraal, A. Limmer, J.M. den Haan, Effective collaboration between marginal metallophilic macrophages and CD8+ dendritic cells in the generation of cytotoxic T cells, Proc. Natl. Acad. Sci. U. S. A. 1 (2010) 216-221.

[54] M.A. Nolte, R. Arens, M. Kraus, M.H. van Oers, G. Kraal, R.A. van Lier, R.E. Mebius, $\mathrm{B}$ cells are crucial for both development and maintenance of the splenic marginal zone, J. Immunol. 6 (2004) 3620-3627.

[55] C. Liu, X.V. Yang, J. Wu, C. Kuei, N.S. Mani, L. Zhang, J. Yu, S.W. Sutton, N. Qin, H. Banie, L. Karlsson, S. Sun, T.W. Lovenberg, Oxysterols direct B-cell migration through EBI2, Nature. 7357 (2011) 519-523.

[56] A.V. Tumanov, S.I. Grivennikov, A.N. Shakhov, S.A. Rybtsov, E.P. Koroleva, J. Takeda, S.A. Nedospasov, D.V. Kuprash, Dissecting the role of lymphotoxin in lymphoid organs by conditional targeting, Immunol. Rev. (2003) 106-116.

[57] Y. You, R.C. Myers, L. Freeberg, J. Foote, J.F. Kearney, L.B. Justement, R.H. Carter, Marginal zone B cells regulate antigen capture by marginal zone macrophages, J. Immunol. 4 (2011) 2172-2181.

[58] Y.S. Kang, Y. Do, H.K. Lee, S.H. Park, C. Cheong, R.M. Lynch, J.M. Loeffler, R.M. Steinman, C.G. Park, A dominant complement fixation pathway for pneumococcal polysaccharides initiated by SIGN-R1 interacting with C1q, Cell. 1 (2006) 47-58.

[59] M.C. Karlsson, R. Guinamard, S. Bolland, M. Sankala, R.M. Steinman, J.V. Ravetch, Macrophages control the retention and trafficking of B lymphocytes in the splenic marginal zone, J. Exp. Med. 2 (2003) 333-340.

[60] T.L. McGaha, Y. Chen, B. Ravishankar, N. van Rooijen, M.C. Karlsson, Marginal zone macrophages suppress innate and adaptive immunity to apoptotic cells in the spleen, Blood. 20 (2011) 5403-5412.

[61] T.L. McGaha, M.C. Karlsson, Apoptotic cell responses in the splenic marginal zone: a paradigm for immunologic reactions to apoptotic antigens with implications for autoimmunity, Immunol. Rev. 1 (2016) 26-43.

[62] G. Hoeffel, J. Chen, Y. Lavin, D. Low, F.F. Almeida, P. See, A.E. Beaudin, J. Lum, I. Low, E.C. Forsberg, M. Poidinger, F. Zolezzi, A. Larbi, L.G. Ng, J.K. Chan, M. Greter, B. 
Becher, I.M. Samokhvalov, M. Merad, F. Ginhoux, C-Myb(+) erythro-myeloid progenitorderived fetal monocytes give rise to adult tissue-resident macrophages, Immunity. 4 (2015) 665-678.

[63] S. Heinz, C. Benner, N. Spann, E. Bertolino, Y.C. Lin, P. Laslo, J.X. Cheng, C. Murre, H. Singh, C.K. Glass, Simple combinations of lineage-determining transcription factors prime cis-regulatory elements required for macrophage and B cell identities, Mol. Cell. 4 (2010) 576-589.

[64] M. Rosas, L.C. Davies, P.J. Giles, C.T. Liao, B. Kharfan, T.C. Stone, V.B. O'Donnell, D.J. Fraser, S.A. Jones, P.R. Taylor, The transcription factor Gata6 links tissue macrophage phenotype and proliferative renewal, Science. 6184 (2014) 645-648.

[65] C. Schneider, S.P. Nobs, M. Kurrer, H. Rehrauer, C. Thiele, M. Kopf, Induction of the nuclear receptor PPAR-gamma by the cytokine GM-CSF is critical for the differentiation of fetal monocytes into alveolar macrophages, Nat. Immunol. 11 (2014) 1026-1037.

[66] M. Kohyama, W. Ise, B.T. Edelson, P.R. Wilker, K. Hildner, C. Mejia, W.A. Frazier, T.L. Murphy, K.M. Murphy, Role for Spi-C in the development of red pulp macrophages and splenic iron homeostasis, Nature. 7227 (2009) 318-321.

[67] T. Tamura, H. Yanai, D. Savitsky, T. Taniguchi, The IRF family transcription factors in immunity and oncogenesis, Annu. Rev. Immunol. (2008) 535-584.

[68] M. Yamamoto, T. Kato, C. Hotta, A. Nishiyama, D. Kurotaki, M. Yoshinari, M. Takami, M. Ichino, M. Nakazawa, T. Matsuyama, R. Kamijo, S. Kitagawa, K. Ozato, T. Tamura, Shared and distinct functions of the transcription factors IRF4 and IRF8 in myeloid cell development, PLoS One. 10 (2011) e25812.

[69] E. Mass, I. Ballesteros, M. Farlik, F. Halbritter, P. Gunther, L. Crozet, C.E. JacomeGalarza, K. Handler, J. Klughammer, Y. Kobayashi, E. Gomez-Perdiguero, J.L. Schultze, M. Beyer, C. Bock, F. Geissmann, Specification of tissue-resident macrophages during organogenesis, Science. 6304 (2016) 10.1126/science.aaf4238. Epub 2016 Aug 4.

[70] M. Haldar, M. Kohyama, A.Y. So, W. Kc, X. Wu, C.G. Briseno, A.T. Satpathy, N.M. Kretzer, H. Arase, N.S. Rajasekaran, L. Wang, T. Egawa, K. Igarashi, D. Baltimore, T.L. Murphy, K.M. Murphy, Heme-mediated SPI-C induction promotes monocyte differentiation into iron-recycling macrophages, Cell. 6 (2014) 1223-1234.

[71] A. Schlitzer, N. McGovern, P. Teo, T. Zelante, K. Atarashi, D. Low, A.W. Ho, P. See, A. Shin, P.S. Wasan, G. Hoeffel, B. Malleret, A. Heiseke, S. Chew, L. Jardine, H.A. Purvis, C.M. Hilkens, J. Tam, M. Poidinger, E.R. Stanley, A.B. Krug, L. Renia, B. Sivasankar, L.G. Ng, M. Collin, P. Ricciardi-Castagnoli, K. Honda, M. Haniffa, F. Ginhoux, IRF4 transcription factor-dependent CD11b+ dendritic cells in human and mouse control mucosal IL-17 cytokine responses, Immunity. 5 (2013) 970-983.

[72] R. Lu, K.L. Medina, D.W. Lancki, H. Singh, IRF-4,8 orchestrate the pre-B-to-B transition in lymphocyte development, Genes Dev. 14 (2003) 1703-1708. 
[73] P. De Togni, J. Goellner, N.H. Ruddle, P.R. Streeter, A. Fick, S. Mariathasan, S.C. Smith, R. Carlson, L.P. Shornick, J. Strauss-Schoenberger, Abnormal development of peripheral lymphoid organs in mice deficient in lymphotoxin, Science. 5159 (1994) 703-707.

[74] P.A. Koni, R. Sacca, P. Lawton, J.L. Browning, N.H. Ruddle, R.A. Flavell, Distinct roles in lymphoid organogenesis for lymphotoxins alpha and beta revealed in lymphotoxin betadeficient mice, Immunity. 4 (1997) 491-500.

[75] S. Hannedouche, J. Zhang, T. Yi, W. Shen, D. Nguyen, J.P. Pereira, D. Guerini, B.U. Baumgarten, S. Roggo, B. Wen, R. Knochenmuss, S. Noel, F. Gessier, L.M. Kelly, M. Vanek, S. Laurent, I. Preuss, C. Miault, I. Christen, R. Karuna, W. Li, D.I. Koo, T. Suply, C. Schmedt, E.C. Peters, R. Falchetto, A. Katopodis, C. Spanka, M.O. Roy, M. Detheux, Y.A. Chen, P.G. Schultz, C.Y. Cho, K. Seuwen, J.G. Cyster, A.W. Sailer, Oxysterols direct immune cell migration via EBI2, Nature. 7357 (2011) 524-527.

[76] J. Emga, H. Kammoun, B. Garcia-Cassani, J. Chesne, SM. Parigi, J-M. Jacob, H-W. Cheng, E. Evren, S. Das, P. Czarnewski, N. Sleiers, F. Melo-Gonzalez, E. Kvedaraite, M. Svensson, E. Scandella, M.R. Hepworth, S. Huber, B. Ludewig, L. Peduto, E.J. Villablanca, H. Veiga-Fernandes, J.P. Pereira, R.A. Flavell, and T. Willinger. Oxysterol Sensing through the Receptor GPR183Promotes the Lymphoid-Tissue-Inducing Function of Innate Lymphoid Cells and Colonic Inflammation, Immunity. 48 (2018). 120-132 


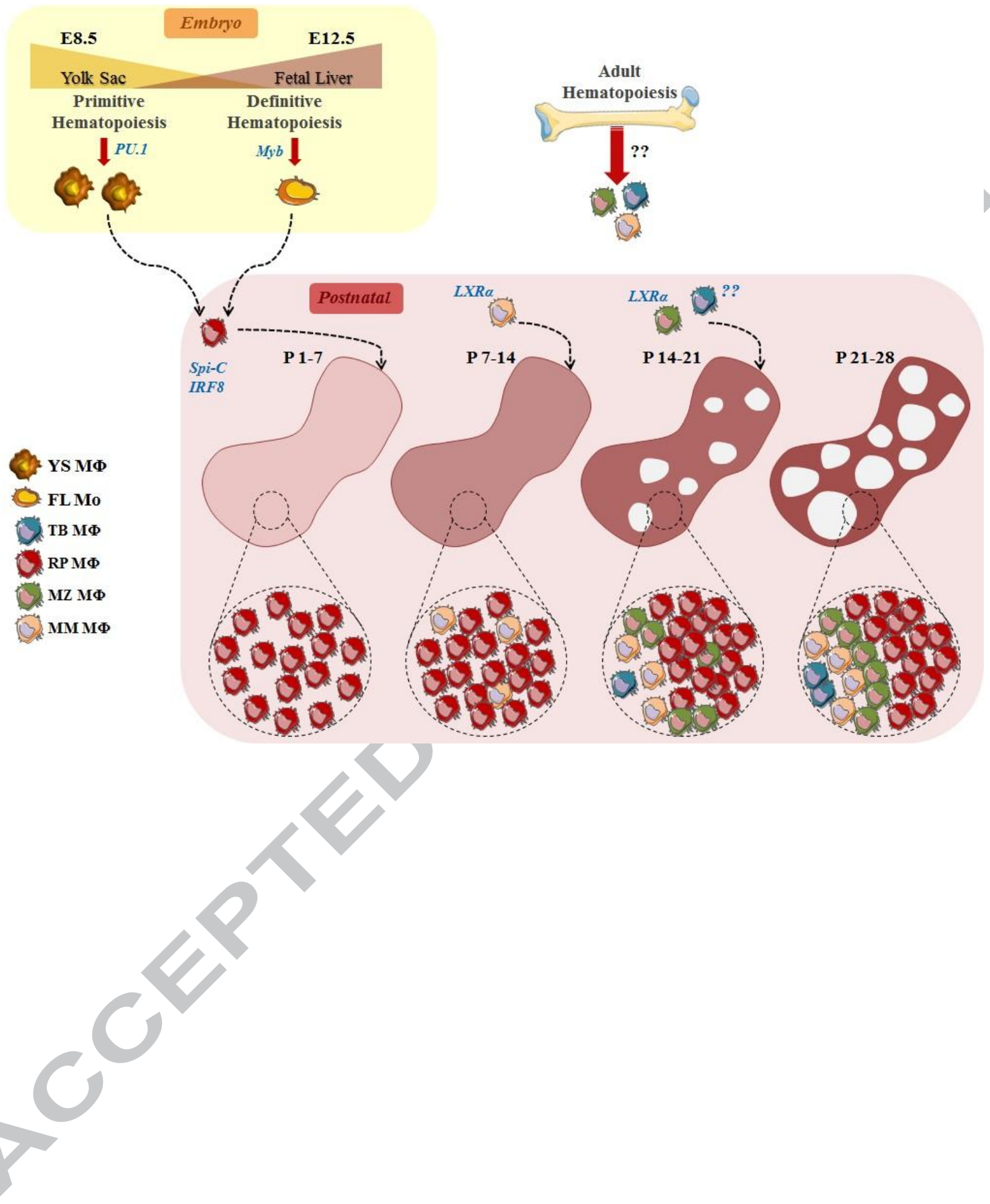




\section{HIGHLIGHTS}

\section{Origin and Specialization of Splenic Macrophages}

\section{Noelia A-Gonzalez and Antonio Castrillo}

- The Spleen is the largest secondary lymphoid organ in the body and the largest filter of the blood.

- The Spleen is crucial for the generation of immune responses to blood-borne antigens and for the clearance of circulating unwanted material.

- At least four different macrophage subsets populate the distinct microanatomical splenic compartments under steady-state conditions.

- Macrophage position and function in their particular splenic microdomains confer them unique phenotypes that are crucial for the defense against blood borne pathogens, clearance of apoptotic cells and iron metabolism.

- Discrete number of transcription factors, including Irf8, Spic and Nr1h3 control the differentiation of red pulp macrophages and marginal zone macrophages. 